\title{
The spectral energy distribution of AO $0235+164$ during two active optical phases
}

\author{
Y. J. Chen ${ }^{1,2}$ and D. R. Jiang ${ }^{1,2}$ \\ 1 Shanghai Astronomical Observatory, Chinese Academy of Sciences, Shanghai 200030, PR China \\ 2 National Astronomical Observatory, Chinese Academy of Sciences, Beijing 100012, PR China
}

Received 2 August 2000 / Accepted 21 June 2001

\begin{abstract}
We investigate the spectral variability of the source AO $0235+164$ during two active phases. We find that the peak frequencies in the Spectral Energy Distributions (SED) are variable with time within a range of an order of magnitude, which does not cause the source to make a transition from Low-energy peaked BL Lac objects to High-energy peaked BL Lac objects. The correlation of peak frequency and peak flux either for an individual outburst or the combination of several individual outbursts is significant. Our research reveals that in many physical properties AO $0235+164$ is much closer to flat-spectrum radio quasars, rather than to High-energy peaked BL Lacs, which in turn implies that Comptonization of external radiation may play quite an important role in $\gamma$-ray emission. There are indications that the observed variability may be due to superposition of intrinsic and propagation effects, which is in agreement with the conclusion of Kraus et al. (1999). We also explore the possible origin of the two outbursts and suggest that intrinsic variability, not including the shock effect, probably accounts for the two outbursts.
\end{abstract}

Key words. galaxies: active - galaxies: BL Lacertae objects: individual: AO 0235+164 - radiation mechanisms: non-thermal

\section{Introduction}

BL Lacertae objects are extragalactic radio sources characterized by strong and rapidly variable nonthermal emission extending from radio to gamma-ray band. The nonthermal continuum is commonly attributed to synchrotron radiation at low frequencies, and inverse Compton radiation at high frequencies emitted in a relativistic jet pointing towards the observer (e.g. Urry et al. 1995). BL Lacs can be divided into two subclasses depending on their Spectral Energy Distribution (SED), namely low-energy peaked BL Lacs (LBL) for objects with synchrotron emission peaking at $\nu_{\mathrm{pk}} \approx 10^{13-14} \mathrm{~Hz}$ and high-energy peaked BL Lacs (HBLs) peaking at $\sim 10^{17-18} \mathrm{~Hz}$ (Padovani \& Giommi 1995). A large sample of blazars was collected by Fossati et al. (1999) to further investigate their spectral energy distributions, from which they found that the correlations between synchrotron peak frequency and luminosity $\left(\nu\right.$ vs. $\left.\nu L_{\nu}\right)$, peak frequency and optical luminosity, and peak frequency and $\gamma$-ray luminosity are significant. Furthermore, with increasing luminosity the synchrotron peak moves to lower frequencies, which implies that

Send offprint requests to: Y. J. Chen,

e-mail: cyj@center.shao.ac.cn radiation losses may play an important role in the radiative process. In contrast, in the present paper we study the changes in the SED of a single source during different outbursts and try to correlate them with the changes in other properties; i.e. rather than sampling a large number of sources once, we sample one source many times.

The radio source AO $0235+164$ was identified by Spinrad \& Smith (1975) as a BL Lac object (a subclass of blazar). The object shows pronounced variability at almost all wavelengths (including strong $\mathrm{GeV} \gamma$-ray emission). These variations are often seen as fast, very intensive flares (Webb \& Smith 1989), of which the two most prominent outbursts took place from late 1975 to early 1976 and from late 1986 to early 1987 (hereafter we refer to them as the 1975 outburst and the 1987 outburst). Takalo et al. (1998) and Kraus et. al. (1999) all suggested that a single physical process cannot explain the observed variability; the former suggested that the effects of microlensing by a foreground galaxy plays an important role and the latter suggested that the observed variability is due to a superposition of intrinsic variability and propagation effects.

In this paper, we use the available multifrequency flux density data from radio to optical wavelength mostly for the two active phases to study the spectral variability of 
AO $0235+164$. In Sect. 2, we give a summary of the multifrequency data and describe the data analysis procedures. We calculate the parameters of simple synchrotron spectra: the peak frequency $\nu_{\mathrm{pk}}$, and the peak flux $\nu F_{\nu}\left(\nu_{\mathrm{pk}}\right)$ for all these epochs. The correlation results of the spectral parameters will be presented in Sect. 3. The general results and discussion can be found in Sect. 4. We conclude with a summary in Sect. 5 .

\section{Multifrequency data for the $\mathbf{1 9 7 5}$ and $\mathbf{1 9 8 7}$ outbursts}

Since the discovery of the radio source AO $0235+164$ in 1972 (Argue et al. 1973), it has undergone rapid, high amplitude variations several times. There are so far five detected outbursts for which observational results have been published. Except for the two outbursts mentioned in Sect. 1, further outbursts occurred in 1979, in 1991 and in 1998 (Roy et al. 2000); of these only three epochs of multifrequency data sets observed during the 1991 outburst were suitable for inclusion in our data analysis. Data sets for two additional epochs, viz. in optical quiescent periods in late 1987 are also included, together with the above three data sets, as a supplement to our further data analysis. We have 33 epochs of different data sets in all for simulation, including 18 collected in 1975, 10 in 1987, 3 in 1991 and 2 in quiescent stage. There are usually $6-9$ observational wavebands for each epoch. Each epoch includes data in at least two radio bands, and two bands in the infrared and optical regions to insure that the peak frequency of $\sim 10^{13} \mathrm{~Hz}$ (Ghisellini et al. 1998) lies within the available wavebands, and that the acquired peak frequencies and energies are more reasonable.

\subsection{The available multifrequency data}

The optical and infrared data came from the observations by Rieke et al. (1976) for the 1975 outburst, Webb \& Smith (1989) for the 1987 outburst, Mead et al. (1990) and Takalo et al. (1998) for the other 5 epoch. The KPNO 1.3-m telescope (Sitko \& Sitko 1991) also made some contribution of infrared and optical data to the 1987 outburst. All of the collected data in infrared and optical wavebands were corrected for galactic extinction as can be found in the corresponding references. In the radio wavebands at $5 \mathrm{GHz}, 8.0 \mathrm{GHz}$ and $14.5 \mathrm{GHz}$ the data were obtained within the Michigan Monitoring Program. As for the data at $22.2 \mathrm{GHz}$ and $36.8 \mathrm{GHz}$, the source has been monitored with the Metsähovi radio telescope since 1980 (Valtaoja et al. 1992). In addition, we obtained data at $10.8 \mathrm{GHz}$ from the Algonquin Radio Observatory (MacLeod et al. 1976), $31.4 \mathrm{GHz}$ data and $85 \mathrm{GHz}$ data from the NRAO 36-foot antenna (Ledden \& Aller 1976). The amount of data at $90 \mathrm{GHz}$ and $230 \mathrm{GHz}$ is relatively small, mainly originating from the 30-m telescope at Pico Veleta (Steppe et al. 1988; Steppe et al. 1993). Above all, we collected data in as many wavebands as possible for each epoch to improve the quality of the fits.
Infrared and optical magnitudes are converted to flux densities for spectral fitting. The optical and infrared flux densities are obtained from the corresponding magnitude using the following expression (Clements et al. 1995),

$f_{\nu}=C_{\nu} \times 10^{6-0.4 m_{\nu}}$

where the coefficient $C_{\nu}$ is the standard Johnson flux density at $m_{\nu}=0$.

\subsection{Multifrequency energy spectral fitting}

Before carrying out the spectral energy fitting, we first grouped the collected multifrequency data according to the observational date, in such a way that due to the rapid variability in optical and infrared wavebands, all multifrequency data for each epoch are grouped to be simultaneous to make sure that the spectral energy distribution truly reflect the intrinsic radiative properties of AO $0235+164$. This has to be done due to the rapid variability in optical and infrared wavebands. At radio wavebands, the variation becomes much slower, and therefore the simultaneity requirement is less stringent (Marscher \& Gear 1985). Centered at the observational date of the optical wavebands, we selected the radio data as close to the center as possible. If the time interval between the observational dates of radio and optical wavebands was over 10 days, the radio data were discarded. If the time interval between two adjacent radio data was less than 20 days at the same frequency, we took the intermediate value using linear interpolation. Above all, the purpose is to make the multifrequency data for each epoch as nearly as possible simultaneous. The specific observing epochs, and the wavebands for each epoch are listed in Table 1.

We constructed the rest-frame spectral energy distributions using the flux densities $F_{\nu}$ of all observational wavebands multiplied by the observed frequency $\nu$ and plot $\nu F_{\nu}$ against $\nu \times(1+z)$ (Sambruna \& Urry 1996). It is known that SEDs clearly show a broad peak between radio and UV-X rays (Fossati et al. 1999). Thus, in order to determine the peak frequency of the synchrotron component for each epoch with an objective procedure, we tried to fit the data points for each epoch using two type of functions, viz., a second degree polynomial (Sambruna \& Urry 1996) and a third degree polynomial (Fossati et al. 1999) respectively to determine which gives the better results. By comparing the results from these two methods, we find that the parabolic fits are not very good, while the fits using a third degree polynomial are much more satisfactory. The reasons may lie in the fact that the optical spectral index is very steep $(-2 \gtrsim \alpha \gtrsim-4)$ (Smith et al. 1987) and the radio spectral index is flatter (Chu et al. 1996); This makes it unlikely that the second degree polynomial fitting result should be very satisfactory. In the following we present all of the spectral fitting results (Fig. 1), of which the left-hand side shows the SEDs of the 1975 outburst and the right-hand one shows the spectral fitting results of the 1987 outburst and the other 5 epochs of data sets. 
Table 1. The observational epochs and observational wavebands for each epoch.

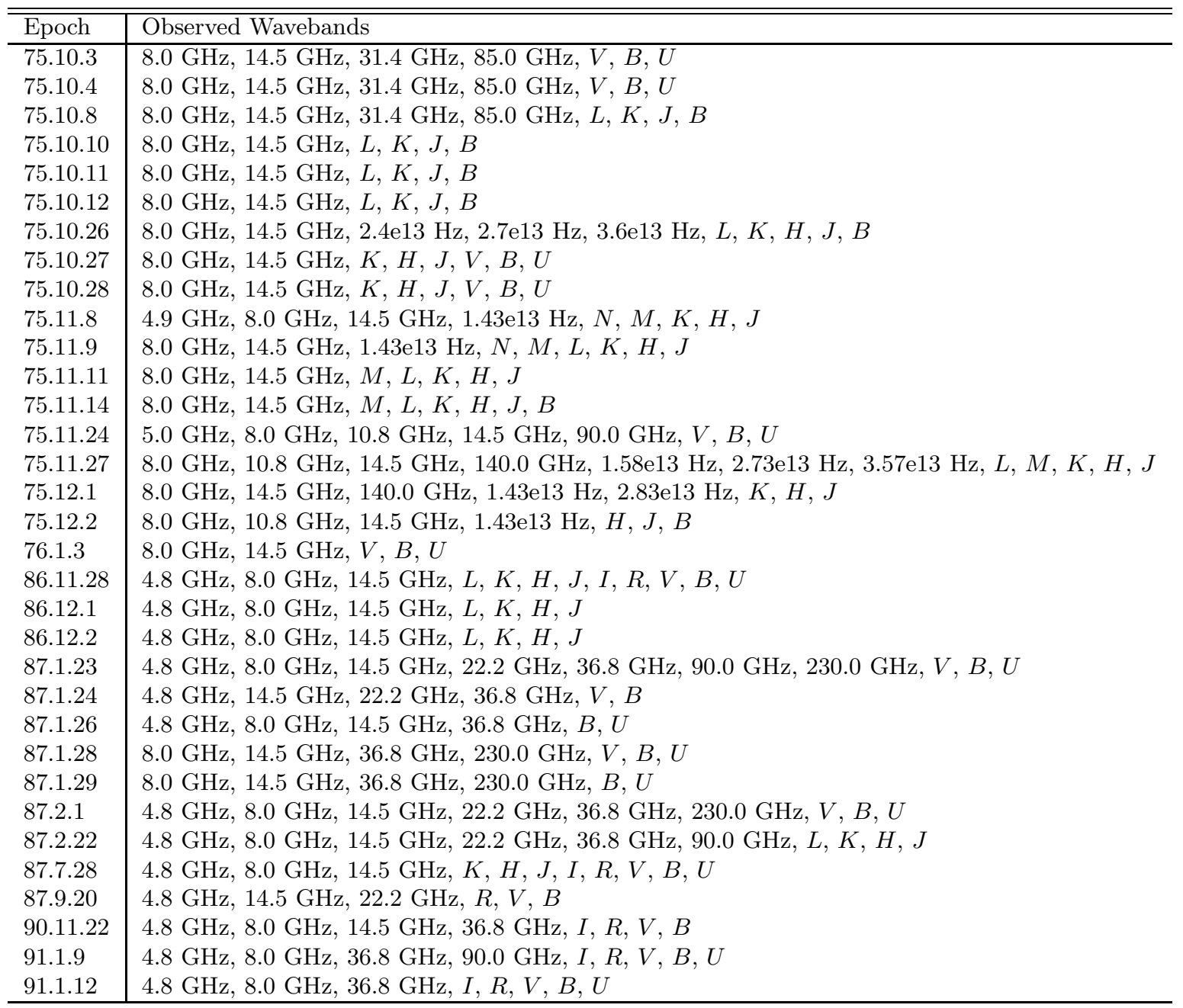

\section{Correlations of spectral quantities with luminosity}

For an individual source, the luminosity distance for all observational epochs is constant so that using $\nu F_{\nu}$ instead of luminosity $\nu L_{\nu}$ will not change the correlation results. Throughout this paper, we refer to the quantity $\nu F_{\nu}$ as peak flux. In addition, all the correlations of physical quantity pairs refer to the linear correlations of the logarithmic values of the corresponding physical quantities.

\subsection{Correlation results for the 1975 outburst}

We obtained peak frequency and peak flux in the course of spectral fitting for each epoch. The distribution of peak flux with peak frequency in SEDs for the 1975 outburst is displayed with the symbol of " $\triangle$ " in the left map of Fig. 2, as we can see, the correlation between $\nu_{\mathrm{pk}}$ and $\nu F_{\nu}\left(\nu_{\mathrm{pk}}\right)$ is apparent. The specific values of correlation coefficient and confidence level are listed in Table 2. A significant correlation of $\nu_{\mathrm{pk}}$ and the luminosity at $8 \mathrm{GHz}$ also exists for the 1975 outburst. Furthermore, there may exist a slight correlation for $\nu_{\mathrm{pk}}$ and optical luminosity at $B$ waveband $\left(\nu F_{\nu}(B)\right)$, but this is not certain.

Except for the pair of $\nu_{\mathrm{pk}}$ vs. $\nu F_{\nu}\left(\nu_{\mathrm{pk}}\right)$ that shows anticorrelation, all the other combinations show positive correlations. A detailed discussion is given in the next section.

\subsection{Correlation results for the 1987 outburst}

In comparison with the 1975 outburst, the number of epochs for which observations were available was smaller, but the observations give a more complete, if somewhat sparse, coverage of the whole outburst.

For the 1987 outburst, the physical quantities of peak frequency and peak flux are well correlated (displayed with symbol "O" in the left map of Fig. 2), yet the correlation of $\nu_{\mathrm{pk}}$ vs. $\nu F_{\nu}(8 \mathrm{GHz})$ is worse. The optical energy in the $B$ waveband is strongly correlated with peak frequency. The detailed correlation parameters are also shown in Table 2.

As was the case for the 1975 outburst, the physical quantities of $\nu_{\mathrm{pk}}$ vs. $\nu F_{\nu}\left(\nu_{\mathrm{pk}}\right)$ show anti-correlation, but the peak frequency and optical luminosity are significantly correlated. On the other hand, luminosity at $8 \mathrm{GHz}$ and peak frequency do not seem to be correlated at all. 

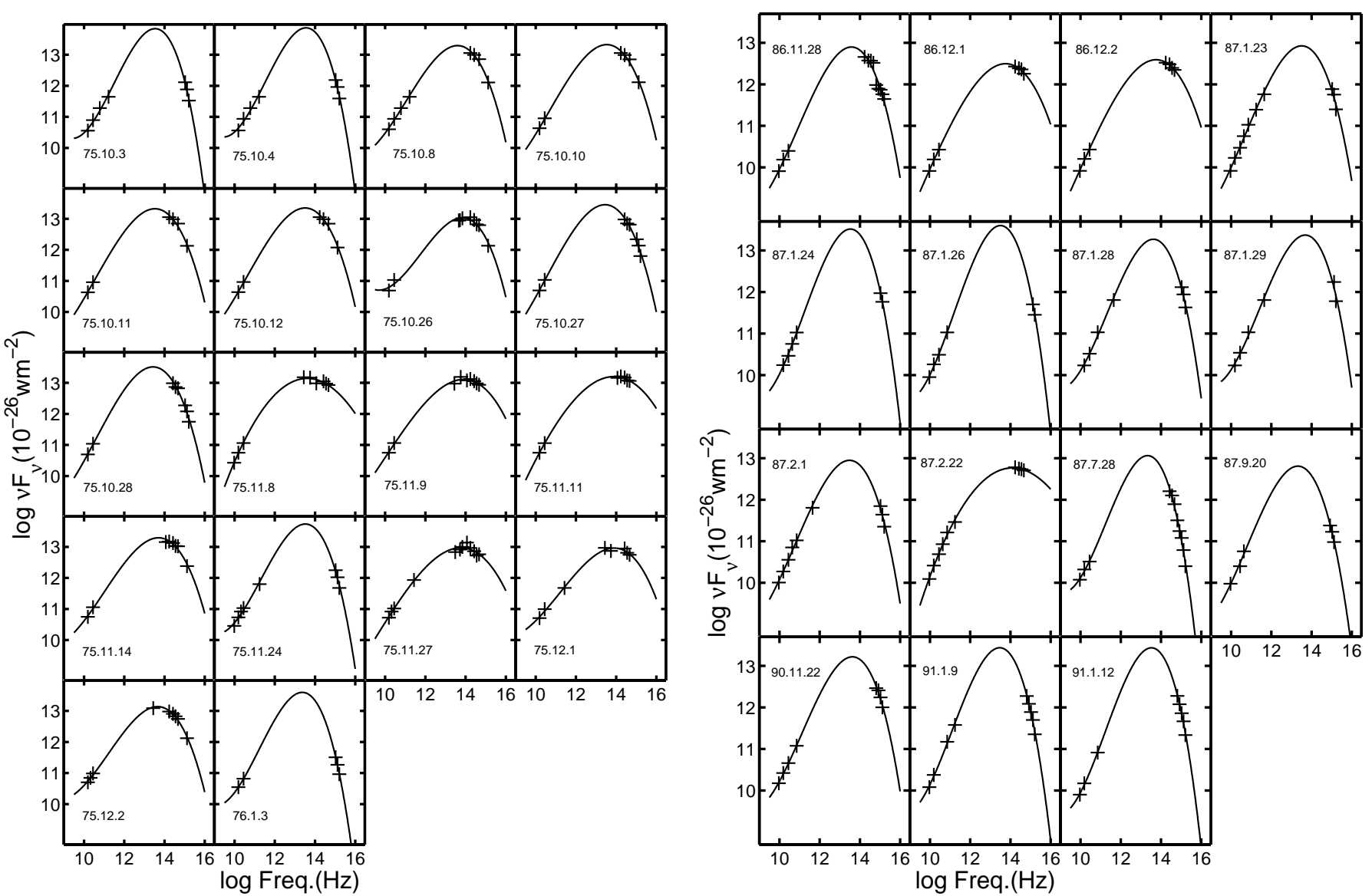

Fig. 1. The intrinsic power $\left(\nu F_{\nu}\right.$, in $\left.10^{-26} \mathrm{wm}^{-2}\right)$ is plotted vs. the rest-frame frequency, $\nu=\nu_{\mathrm{obs}} \times(1+z)$. Left map: fitting results for all epochs of the 1975 outburst; right map: fitting results for all epochs of the 1987 outburst as well as the other 5 epoch of multi-frequency data.

It should be noted that the correlation of $\nu_{\mathrm{pk}}$ vs. radio energy at $8 \mathrm{GHz}$ is strong for the 1975 outburst, but weak for the 1987 outburst.

\subsection{Correlation results for all epochs}

We combined the fitting results of all epochs, including the 1975 and 1987 outbursts together with the other 5 epochs, and then present the general results (see Fig. 2). We can see from Table 2 that the correlation of $\nu_{\mathrm{pk}}$ vs. optical luminosity in the $B$ waveband is very strong, and there appears to be no correlation of $\nu_{\mathrm{pk}}$ vs. $\nu F_{\nu}(8 \mathrm{GHz})$. After combination, the correlation of $\nu_{\mathrm{pk}}$ vs. $\nu F_{\nu}\left(\nu_{\mathrm{pk}}\right)$ becomes weaker but it still remains fairly strong, let alone the correlation for both individual outbursts. In short, the peak frequency in SEDs is well correlated with the optical flux in the $B$ band and the peak flux. Yet the correlation between peak frequency and luminosity at $8 \mathrm{GHz}$ is not significant.

\section{Results and discussion}

Blazars are characterized by large amplitude and fast variability, indicating that the electron distribution is rapidly changing or the orientation of the emitting jet is varying with time. There appears to be a more or less continuous sequence of spectral properties of different objects, $\mathrm{HBL} \rightarrow \mathrm{LBL} \rightarrow \mathrm{FSRQs}$ (flat spectrum radio quasars), characterized by increasing $\gamma$-ray luminosity, increasing dominance of the energy output in the $\gamma$-ray component over the synchrotron component, and a shift of the peaks in the $\nu F_{\nu}$ spectra of both components towards lower energies. This sequence can be understood in terms of increasing dominance of the external comptonization (EC) over the synchrotron self-Compton (SSC) mechanism (e.g., Fossati et al. 1997). These different types of object may show different behaviour in the evolution of the synchrotron peak in their SEDs, and therefore can be used as a diagnostic to determine the dominant radiation mechanism in these objects (Böttcher 1999). Moreover the changes of the electron spectrum, and physical parameters such as magnetic field strength inside the jet can also affect the evolution behaviour of the synchrotron peak in SEDs (Mastichiadis \& Kirk 1997; Petry et al. 2000). In addition, spectral variability may also involve some other factors such as the size of the emitting region, the orientation variation of the emitting jet and possible shock formation in jet as well (Chiaberge \& Ghisellini 1999; Villata \& Raiteri 1999; Kirk et al. 1998). Obviously a shock forming in the jet will change the particle energy distributions 

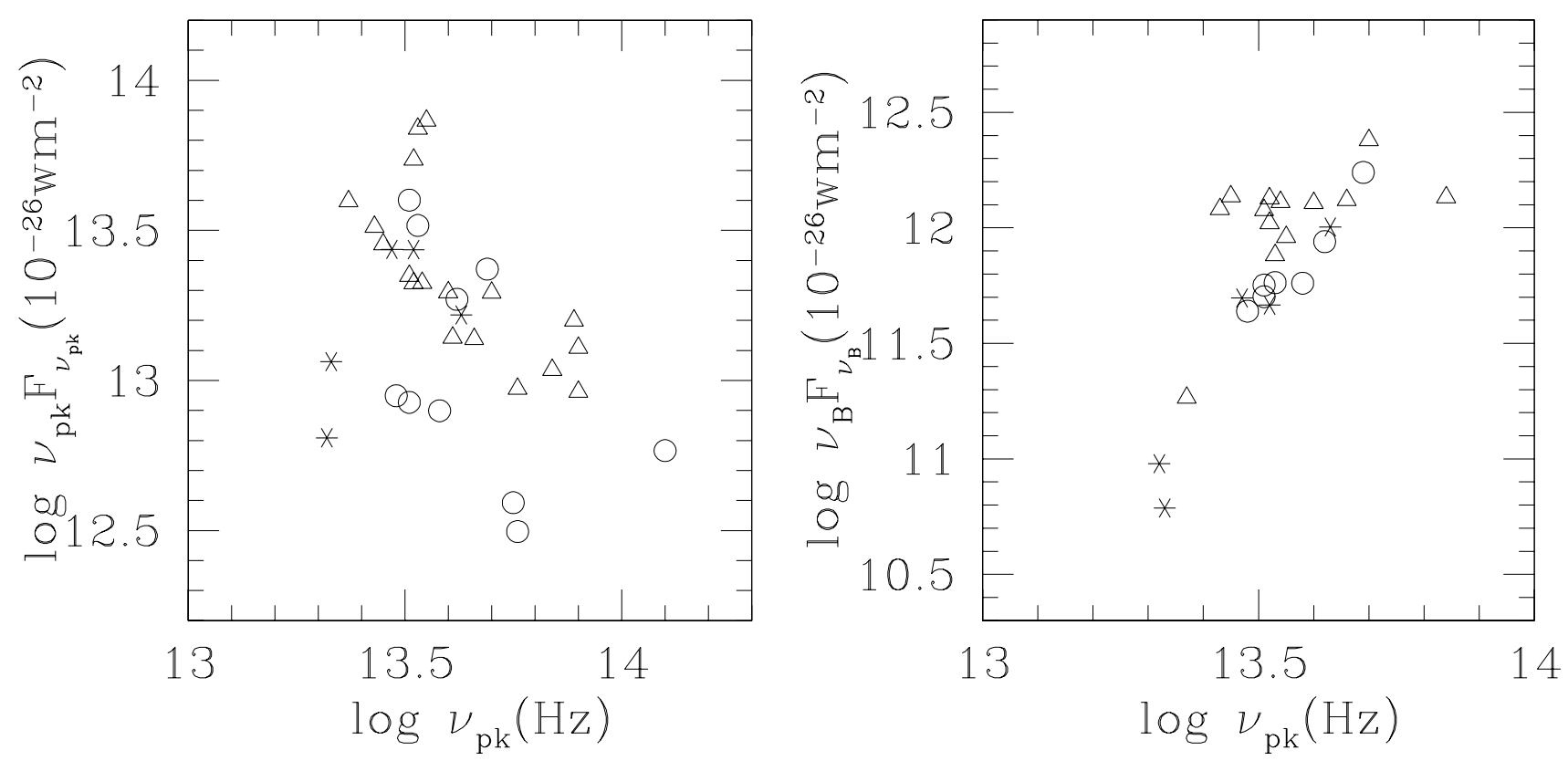

Fig. 2. Left map: distribution of peak frequency and peak flux for all epochs: " $\triangle$ " stands for the 1975 outburst, "O" stands for the 1987 outburst, "* " for the other epochs; right map: distribution of optical energy in the $B$ waveband with peak frequency (symbols are the same as on the left).

Table 2. Correlation coefficients and corresponding confidence levels for all combinations.

\begin{tabular}{c|c|c|c|c|c|c}
\hline \hline Correlated Quantities & \multicolumn{2}{|c|}{1975} & \multicolumn{2}{c|}{1987} & \multicolumn{2}{c}{ All epochs } \\
\cline { 2 - 7 } & $r^{\text {a }}$ & Confidence & $r$ & Confidence & $r$ & Confidence \\
\hline$\nu_{\mathrm{pk}}-\nu F_{\nu}(\mathrm{pk})$ & -0.806 & $8.93 \mathrm{e}-4$ & -0.608 & $6.82 \mathrm{e}-2$ & -0.422 & $1.70 \mathrm{e}-2$ \\
$\nu_{\mathrm{pk}}-\nu F_{\nu}(8 \mathrm{GHz})$ & 0.567 & $1.94 \mathrm{e}-2$ & -0.207 & 0.534 & 0.248 & $1.60 \mathrm{e}-1$ \\
$\nu_{\mathrm{pk}}-\nu F_{\nu}(B)$ & 0.451 & $1.18 \mathrm{e}-1$ & 0.955 & $1.93 \mathrm{e}-2$ & 0.628 & $2.08 \mathrm{e}-3$ \\
\hline
\end{tabular}

a $r$ is the correlation coefficient.

and the orientation variation of the emitting region will change beaming factor so that the spectral variability will show different behaviour in both cases (Villata \& Raiteri 1999; Kirk et al. 1998).

\subsection{Spectral energy distribution of $A O 0235+164$}

In terms of the correlation coefficients and corresponding confidence values in Table 2 , for the radio source of $\mathrm{AO}$ $0235+164$, there is a significant inverse correlation of peak frequency and peak flux both for individual outbursts and for the combination of the two outbursts with the other 5 epoch of SEDs. The synchrotron peak frequencies of about $10^{13-14} \mathrm{~Hz}$ (see Fig. 2) of the source are much closer to those of FSRQs (about $10^{12-13} \mathrm{~Hz}$; Böttcher 1999) than to those of HBLs (about $10^{17-18} \mathrm{~Hz}$ ). Böttcher (1999) pointed out that if the $\gamma$-ray emission of FSRQs is produced by Comptonization of external radiation which dominates the radiative energy loss rate of relativistic electrons in blobs, then the observed peak of the synchrotron spectrum component depends on the bulk Lorentz factor $\Gamma$ and the external photon density $u_{\text {ext }}$ as

$\nu_{\mathrm{pk}} \propto \dot{\gamma}_{\mathrm{acc}} B^{\prime} u_{\mathrm{ext}}^{-1} \Gamma^{-1}$
$L_{\mathrm{pk}} \propto \dot{\gamma}_{\mathrm{acc}} B^{\prime 2} u_{\mathrm{ext}}^{-1} \Gamma^{2}$

where $B^{\prime}$ and $\dot{\gamma}_{\text {acc }}$ are the magnetic field strength in the comoving frame and electron acceleration rate respectively. From the above two equations we can have the expression as

$\nu L_{\nu}\left(\nu_{\mathrm{pk}}\right) \propto \nu_{\mathrm{pk}}^{-1}$

if $B^{\prime}$ remains constant or independent of the other quantities. The synchrotron peak is therefore expected to shift toward lower frequencies with increasing Lorentz factor while the peak density generally increases in the $\gamma$-ray flaring stage. AO 0235+164 is a low-peaked BL Lac, located at intermediate position in the sequence of subclasses of Blazar mentioned above. Nuclear emission features were detected by Cohen et al. (1987) in the optical spectrum of the source, showing broad permitted Mg II and narrow forbidden lines, which is a sign of the presence of an ambient medium. This may indicate that AO $0235+164$ fulfills the prerequisite of producing enough soft photons as seeds for inverse Compton scattering. Madejski et al. (1996) also pointed out that AO $0235+164$ has all the characteristics of optically violent variable (OVV) quasars (including the $\mathrm{GeV}$ emission), except that it shows weaker 
emission lines. They suggested that the SSC interpretation of $\gamma$-ray production may be challenged by the fact that the fast variability in the soft X-ray band is not accompanied by variability in $\gamma$-rays. All these points mentioned above imply that AO $0235+164$ in many physical properties is much closer to FSRQs rather than HBLs, which in turn indicates that Comptonization of external radiation may play a quite important role in $\gamma$-ray emission in the source. Therefore it is feasible to interpret the evolution behaviour of synchrotron peak of AO $0235+164$ by a change of Lorentz factor.

In the course of linear fitting to the distribution of peak frequency and peak flux, we also obtained the coefficients of all polynomials. For the 1975 outburst, we have

$\log \left[\nu F_{\nu}\left(\nu_{\mathrm{pk}}\right)\right] \approx 29.43-1.18 \log \nu_{\mathrm{pk}}$

And for the 1987 outburst, the relation can be expressed as

$\log \left[\nu F_{\nu}\left(\nu_{\mathrm{pk}}\right)\right] \approx 26.75-1.00 \log \nu_{\mathrm{pk}}$

By comparing Eq. (5) with Eq. (6), we find that the values of the coefficient of the second term are very close to each other in the above two equations, essentially within the range of fitting errors. Therefore from Eqs. (5) and (6) we could have the same expression as Eq. (4), that is, $\nu F_{\nu}\left(\nu_{\mathrm{pk}}\right) \propto \nu_{\mathrm{pk}}^{-1}$. This may in turn be a strong support for the conclusion by Böttcher (1999), and also indicates that Comptonization of external photons probably plays an important role in $\gamma$-ray emission in the two outbursts.

It is noticeable that an opposite correlation behavior was found in the BL Lac object Mkn 501 (e.g. Petry et al. 2000) and Mkn 421 (Fossati et al. 2000), in which peak flux increases with peak frequency. Both of the two sources belong to HBLs that peak at X-ray bands. As pointed out by Böttcher (1999), the broad-line regions of these objects surrounding the central engine are weak or absent and the isotropic luminosity of the central accretion disk is generally weaker than in quasars, implying that the external soft photon field is much weaker than in FSRQs and hence negligible compared to the intrinsic synchrotron radiation field; Moreover, the Compton scattering events near the $\nu F_{\nu}$ peak of the $\gamma$-ray spectrum most probably occur in the extreme klein-Nishina regime, thus rendering Compton cooling rather inefficient. However, the fact that the emission lines, although not very strong, are present and that $\mathrm{GeV}$ rather than $\mathrm{TeV} \gamma$-ray emission is detected in AO 0235+164, at least implies that an external comptonization interpretation of $\gamma$-ray emission is possible. In Mkn 421 and Mkn 501 the hardening of the electron spectrum and/or the change of the maximum energy of the electron injection spectrum could effectively explain the evolution behaviour of the synchrotron peak of these objects (Petry et al. 2000; Mastichiadis \& Kirk 1997).

Another of the most common used interpretations for Mkn 501 is the change of jet orientation probably due to the orbital motion of the parent black hole in a binary black hole system (BBHS) and hence leading to a change of the relativistic beaming factor, which causes a large shift of the X-ray peak of the SED from 10-20 keV in 1996 to $100-200 \mathrm{keV}$ in 1997 (Villata \& Raiteri 1999; Rieger \& Mannheim 2000). Phenomena in Mkn 501 such as a discontinuity of the synchrotron spectrum, or exceptional brightening and hardening of the X-ray spectrum without a substantial change at lower frequencies have not been observed in AO $0235+164$. In addition smallscale distortions such as are seen in the VLBI map of Mkn 501 (Giovannini et al. 1998) which is further evidence for BBHS model are also not observed in high-resolution images of AO $0235+164$. This may merely imply that it is not the change of jet orientation which is mainly responsible for the spectral variability in $\mathrm{AO} 0235+164$.

A peculiar consequence of the shock model is that the turnover flux density increases or essentially remain constant while the turnover frequency decreases in the rising and peaking stage (Marscher 1985; Valtaoja et al. 1992). This property can be used to decide if the two outbursts can be caused by the formation and propagation of shock waves. We used the method proposed by Landau et al. (1986) to fit a continuous curve to the spectral flux density distribution with a second degree polynomial. The final result is shown in Fig. 3, in which the flux density at the turnover frequency generally increases with turnover frequency. Since most of the obtained data lie in the peaking and rising stage (see Fig. 4), It seems that the shock model doesn't correspond to the scenario of the two outbursts in AO $0235+164$.

In addition, we can see from Fig. 2 that the peaking frequency and peaking energy in SEDs are variable within a range of no more than an order of magnitude. This implies that for a low energy peaked BL Lac object, the peak frequency, although variable, will not leave this range, and the object will not appear to be a high energy-peaked BL Lac object.

\subsection{Comparison between the 1975 outburst and the 1987 outburst}

From Table 2 we can find that for the 1975 outburst, the peak frequency shows a positive correlation with radio energy at $8 \mathrm{GHz}$ and with optical energy in the $B$ waveband (although not a very strong one for $\nu_{\mathrm{pk}}$ vs. $\nu F_{\nu}(B)$ due to the fact that most epochs lie near to the peaking stage), yet the peak frequency shows an inverse correlation with peak flux. For the 1987 outburst, the situation is similar. The difference lies in this that the peak frequency is well correlated with optical energy, but essentially not correlated with radio energy. There is some difficulty in explaining such a scenario satisfactorily. Since the spectral shape - the spectral index, changes with time, it is not necessary that flux densities at all frequencies of synchrotron component are increasing or decreasing in concert, and one can even have cases in which at certain frequencies the fluxes decrease while at other frequencies the fluxes increase. Different sources may show different behavior, as is seen in objects PKS $0528+134$ and 3C 273 


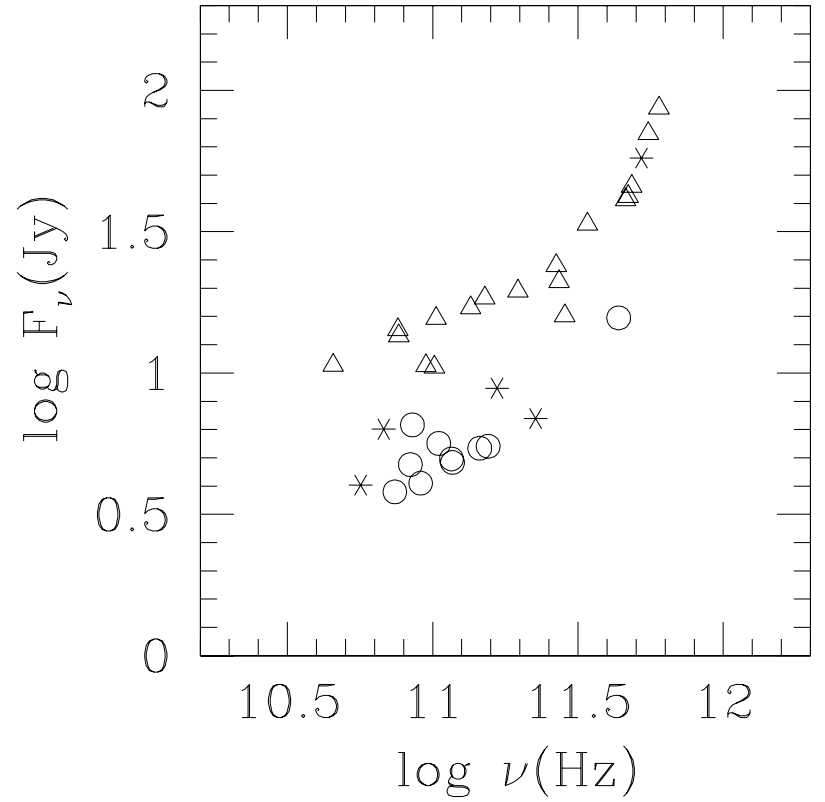

Fig. 3. The distribution of turnover flux density with turnover frequency. Symbols are the same as Fig. 2.

(see Figs. 1 and 2 in Böttcher 1999). This will be the subject of further study.

Ghisellini et al. (1998) pointed out that the different SEDs may reflect different predominant electron cooling mechanisms. As mentioned above AO $0235+164$ is an EGRET source, and most of its power may be emitted in the $\gamma$-ray band through inverse Compton Scattering, especially during the stage of $\gamma$-ray flaring (probably due to the change of Lorentz factor). At this point the two outbursts seem to be very similar. However, distinctions are also present. The correlation difference between peak frequency and flux density at $8 \mathrm{GHz}$ for the two outbursts implies that there must be something else that works for one or both of them. Furthermore, since the acquired turnover frequency distribution for the 1975 outburst is relatively higher than that for the 1987 outburst (see Fig. 3), we suggest that the emitting region for the 1975 outburst is more compact than that for the 1987 outburst so that the peak frequency distribution for the 1975 outburst may shift slightly to a lower frequency and become relatively lower than that for the 1987 outburst (see the left map of Fig. 2). In addition, we also find that the 1975 outburst has a larger amplitude of variation both in optical and in radio bands (see Fig. 4 in our present paper and also Fig. 4 in Chen et al. 1999), which probably means a more violent flaring in the 1975 outburst.

The broadband (radio - $\gamma$-ray) emission from blazars is most probably emitted via nonthermal synchrotron radiation and Comptonization of soft photons by energetic particles in relativistic jet, and the total emitted power for a single electron is proportional to the square of its energy, that is, $\frac{\mathrm{d} \gamma}{\mathrm{d} t} \propto \gamma^{2}$, for both radiative processes. Therefore it is easy to turn high-energy electrons into low energy electrons, and this will lead to softening of the particle

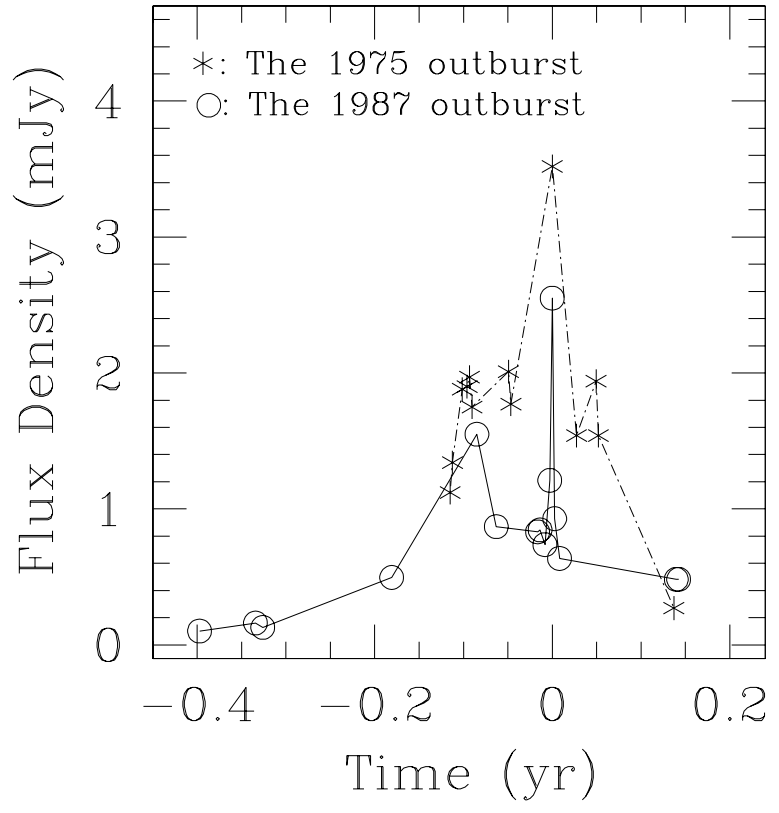

Fig. 4. Flux density variation with time for the 1975 outburst and the 1987 outburst.

spectrum. This will facilitate the movement of synchrotron peaks in SEDs to lower waveband, and hence more easily result in an inverse correlation between the two physical quantities. Since photons produced in different parts of the source have different travel times, the observed spectrum is produced by the electron distribution at different stages of evolution. The shape of the light curve in the optical band (see Fig. 4) and in X-ray band observed in 1993 (see Fig. 1 in Madejski et al. 1996), showing quasisymmetric behaviour, suggests that the cooling time of the emitting electrons is shorter than the light crossing time (Chiaberge \& Ghisellini 1999). This probably implies that the observed variability is due to superposition of intrinsic and propagation effects which is consistent with Kraus et al. (1999).

In addition, since flux is generally thought to come from progressively larger regions as frequency falls, a lag is expected between the optical and the radio variations. For this reason, we use the method of discrete correlation function (DCF) (Edelson \& Krolik 1989) and find that the radio flux density variation at $8 \mathrm{GHz}$ and optical variation in the $B$ waveband are correlated with a delay of $\sim 20$ days for the 1975 outburst and $\sim 80$ days for the 1987 outburst, which can well explain the correlation results shown in Table 2 viz. that peak frequency is well correlated with flux density at $8 \mathrm{GHz}$ for the 1975 outburst but not for the 1987 outburst.

\section{Summary}

In this paper we collect 33 epochs of multifrequency data sets of the radio source AO $0235+164$, including 18 sets for the 1975 outburst and 10 for the 1987 outburst together with 5 data sets at other times. A third degree polynomial fitting to all these data sets is carried out to obtain 
the peak frequency and energy in SEDs. By analysing the correlation of peak frequency with peak flux, we find that:

1. The peak frequency of AO $0235+164$ is variable with time within a range of an order of magnitude. All the peak frequencies for the 33 SEDs are within the peak frequency range of $\mathrm{LBL}$, which is used to define the subclass of BL Lacs (Padovani \& Giommi 1995);

2. The correlation of peak frequency and peak flux either for an individual outburst or the combination of several individual outbursts is significant and is well represented by $\nu F_{\nu}\left(\nu_{\mathrm{pk}}\right) \propto \nu_{\mathrm{pk}}^{-1}$. This can be interpreted well by a change of Lorentz factor in objects, in which Comptonization of external radiation dominates $\gamma$-ray emission (Böttcher 1999). AO 0235+164 in many physical properties is much closer to FSRQs rather than HBLs, which in turn implies that Comptonization of external radiation may play important role in $\gamma$-ray emission in the source. Intrinsic variability not including the shock effect is probably the primary origin of the two outbursts. Some observational results indicate that the observed variability is probably due to superposition of intrinsic and propagation effects which is consistent with the conclusion of Kraus et al. (1999);

3. A comparison between the 1975 outburst and the 1987 outburst reveals that the two outbursts have a similar flaring time scale, and the 1975 outburst has a larger amplitude of variation and relatively higher turnover frequency distribution than that for the 1987 outburst. This may indicate that the 1975 outburst is more violent and the corresponding emitting region is more compact.

Acknowledgements. We would like to thank the anonymous referee(s) for useful suggestions and opinions for correction. Also we shall appreciate a lot of help in english writing from Dr. J. Katgert and Dr. W. P. Lin. This research has made use of data from the University of Michigan Radio Astronomy Observatory which is supported by the National Science Foundation and by funds the University of Michigan. The work is supported by Chinese NKBRSF grant G19990754.

\section{References}

Argue, A. N., Kenworthy, C. M., \& Stewart, P. M. 1973, ApJ, 14, L99

Böttcher, M. 1999, ApJ, 515, L21

Chiaberge, M., \& Ghisellini, G. 1999, MNRAS, 306, 551
Chen, Y. J., Zhang, F. J., \& Sjouwerman, L. O. 1999, ApSS, 266, 495

Chu, H. S., Bååth, L. B., Zhang, F. J., Nicholson, G., \& Rantakyrö, F. T. 1996, A\&A, 307, 15

Cohen, R. D., Smith, H. E., Junkkarinen, V. T., \& Burbidge, E. M. 1987, ApJ, 318, 577

Edelson, R. A., \& Krolik, J. H. 1988, ApJ, 333, 646

Fossati, G., Celotti, A., Ghisellini, G., \& Maraschi, L. 1997, ApJ, 289, 136

Fossati, G., Maraschi, L., Celotti, A., et al. 1999, MNRAS, 299, 433

Fossati, G., Celotti, A., Chiaberge, M., et al. 2000, ApJ, 541, 166

Ghisellini, G., Celotti, A., Fossati, G., Maraschi, L., \& Comastri, A. 1998, MNRAS, 301, 451

Giovannini, G., Cotton, W. D., Feretti, L., Lara, L., \& Venturi, T. 1998, in Proc. 32nd COSPAR Scientific Assembly. Adv. Space Res., in press

Kirk, J. G., Rieger, F. M., \& Mastichiadis, A. 1998, A\&A, 333, 452

Kraus, A., Quirrenbach, A., Lobanov, A. P., et al. 1999, A\&A, 344,807

Ledden, J. E., \& Aller, H. D. 1976, Nature, 260, 752

MacLeod, J. M., Andrew, B. H., \& Harvey, G. A. 1976, Nature, 260, 751

Madejski, G., Takahashi, T., Tashiro, M., et al. 1996, ApJ, 459, 156

Marscher, A. P., \& Gear, W. K. 1985, ApJ, 298, 114

Mastichiadis, A., \& Kirk, J. G. 1997, A\&A, 320, 19

Mead, A. R. G., Ballard, K. R., Brand, P. W. J. L., et al. 1990, A\&AS, 83, 183

Padovani, P., \& Giommi, P. 1995, ApJ, 444, 567

Petry, D., Böttcher, M., Connaughton, V., et al. 2000, ApJ, 536, 742

Rieger, F. M., \& Mannheim, K. 2000, A\&A, 359, 948

Rieke, G. H., Grasdalen, G. L., Kinman, T. D., et al. 1976, Nature, 160, 754

Roy, M., Papadakis, I. E., Ramos-Colóm, E., et al. 2000, ApJ, 545,758

Sambruna, R. M., Maraschi, L., \& Urry, C. M. 1996, ApJ, 463, 444

Sitko, M., \& Sitko, A. 1991, PASP, 103, 160

Smith, P. X., Balonek, T. J., Elston, R., \& Heckert, P. A. 1987, ApJS, 64, 459

Spinrad, H., \& Smith, H. E. 1975, ApJ, 201, 275

Steppe, H., Salter, C. J., Chini, R., et al. 1988, A\&AS, 75, 317

Steppe, H., Paubert, G., Sievers, A., et al. 1993, A\&AS, 102, 611

Takalo, L. O., Sillanpää, A., Valtaoja, E., et al. 1998, A\&AS, 129,577

Valtaoja, E., Teräsranta, H., Urpo, S., et al. 1992, A\&A, 254 71

Villata, M., \& Raiteri, C. M. 1999, A\&A, 347, 30

Webb, J. R., \& Smith, A. G. 1989, A\&A, 220, 65 